\title{
Efficient Modeling of Embedded Systems Using Computer-Aided Recoding
}

\author{
Rainer Dömer \\ Center for Embedded Computer Systems \\ University of California, Irvine \\ doemer@uci.edu
}

\begin{abstract}
The design of embedded computing systems faces a serious productivity gap due to the increasing complexity of their hardware and software components. One solution to address this problem is the modeling at higher levels of abstraction. However, writing proper executable system models is challenging, error-prone, and very time-consuming.

This tutorial outlines a novel modeling technique called computer-aided recoding which automates the process of specifying and modeling embedded systems by use of advanced computer-aided design (CAD) techniques. Using a designer-controlled approach with automated source code transformations, computer-aided recoding can derive an executable parallel system model directly from available sequential reference code. As a result, system modeling is streamlined, enabling a shorter design time and higher productivity.
\end{abstract}

Keywords: Embedded System Design, Specification and Modeling, ComputerAided Recoding.

\section{Introduction}

Embedded computing systems, such as video-enabled mobile phones and reliable medical devices, are omnipresent and pervasive in our everyday life. The design of these systems, however, is very challenging due to hard design constraints, including strict timing, multi-core functionality, low power, low price, and short time-to-market. Moreover, we face a growing design productivity gap due to the increasing complexity of the embedded hardware and software components.

The International Technology Roadmap for Semiconductors (ITRS) lists modeling at higher levels of abstraction as key to overcome the productivity gap [1]. Much like the quality of an architectural blue-print determines the quality of the resulting building, the model of an embedded system is the key to its successful implementation. However, writing proper executable system models is challenging, error-prone, and very time-consuming. For a simple MP3 decoder application, our experiments have shown that more than $90 \%$ of the overall design time was spent in creating and editing the system model [2]. Other studies also confirm that the model specification phase is a serious bottleneck in embedded system design. 


\section{Computer-Aided Recoding}

In this tutorial, we present computer-aided recoding, a new specification and modeling technique that automates various steps in the process of writing embedded system models. Computer-aided recoding is a designer-controlled approach that relies on automated source code transformations available to the system designer in form of a "smart" integrated development environment [2]. In this approach, the designer makes the decisions, whereas the tool automatically transforms the source code.

Specifically, we discuss several types of source code transformations, including code and data partitioning to create parallel and flexible system models [3], pointer recoding to eliminate unwanted pointers in given $\mathrm{C}$ reference code [4], creation of structural hierarchy to properly organize the initially unstructured "flat" application code [5], and exposing potential parallelism and creating explicit communication and synchronization in the system model [6]. Using our prototype implementation of an interactive source recoder [2], we provide experimental results that demonstrate the effectiveness of the approach and show significant gains in design productivity.

\section{Conclusion}

Computer-aided recoding can derive an executable parallel system model directly from available sequential reference code. Automatic source code transformations relieve the system designer from complex code analysis and tedious coding tasks, allowing uninterrupted focus on system modeling and design space exploration. As a result, modeling writing is streamlined, enabling a shorter design time and higher productivity, as well as quality improvements in the end design.

\section{References}

1. International Semiconductor Industry Association: International Technology Roadmap for Semiconductors (ITRS) (2007), http: / / www . itrs. net

2. Chandraiah, P., Dömer, R.: An Interactive Model Re-Coder for Efficient SoC Specification. In: Rettberg, A., Zanella, M., Dömer, R., Gerstlauer, A., Rammig, F. (eds.) Proceedings of the International Embedded Systems Symposium, Embedded System Design: Topics, Techniques and Trends. Springer, Irvine (2007)

3. Chandraiah, P., Dömer, R.: Designer-Controlled Generation of Parallel and Flexible Heterogeneous MPSoC Specification. In: Proceedings of the Design Automation Conference 2007, San Diego, California (June 2007)

4. Chandraiah, P., Dömer, R.: Pointer Re-coding for Creating Definitive MPSoC Models. In: Proceedings of the International Conference on Hardware/Software Codesign and System Synthesis, Salzburg, Austria (September 2007)

5. Chandraiah, P., Dömer, R.: Automatic Re-coding of Reference Code into Structured and Analyzable SoC Models. In: Proceedings of the Asia and South Pacific Design Automation Conference 2008, Seoul, Korea (January 2008)

6. Chandraiah, P., Dömer, R.: Code and Data Structure Partitioning for Parallel and Flexible MPSoC Specification Using Designer-Controlled Re-Coding. IEEE Transactions on Computer-Aided Design of Integrated Circuits and Systems 27(6), 1078-1090 (2008) 